\title{
Introduction to the Special Issue on Translation
}

\author{
Douglas DeWitt and Donald S. Prough
}

D ESPITE THE NUMEROUS promising neuroprotective agents identified in experimental traumatic brain injury (TBI) studies, none has shown meaningful improvement in long-term outcome in hundreds of clinical trials conducted over the last few decades. ${ }^{1-3}$ Included among the myriad reasons for the limited translational success of pharmacological therapies for TBI are major differences in the types and times of collection of outcome variables between clinical and pre-clinical studies ${ }^{4}$ and the limited number of pre-clinical studies involving multiple TBI models an$\mathrm{d} /$ or multiple species. ${ }^{5,6}$ In addition to these and other limitations in pre-clinical therapy testing, the validity of many clinical trials has been reduced by inadvertent bias, ${ }^{7}$ and the relatively large number of single-center ${ }^{1}$ and underpowered clinical trials. ${ }^{8,9}$ These and other potential reasons for the failure of candidate therapies to translate to successful randomized controlled trials (RCTs) and clinical benefits in patients with TBI are discussed in greater detail in several recent reviews. ${ }^{4,10-13}$

This special issue on translation is intended to address some of the challenges associated with the successful transition of potentially effective pharmacological therapies from laboratory studies to clinical practice.

Kochanek and colleagues describe traditional and novel approaches to the identification and pre-clinical development of effective therapies for TBI such as Operation Brain Trauma Therapy (OBTT), a multi-center consortium that has provided pre-clinical testing of 12 potential therapies for TBI in three rodent TBI models. Also, they suggest that the incorporation of biomarkers into preclinical testing could enhance the choice and assessment of target engagements of potential therapies, that drug combinations may be more effective in some patients, and that precision medicine approaches may improve therapeutic efficacy by linking specific therapies to specific patients by more accurate TBI classification and/or pathophysiological phenotyping. Finally, they describe Targeted Evaluation, Action, and Monitoring of TBI (TEAM TBI), a group of investigators at the University of Pittsburgh Brain Trauma Research Center who are using symptomatic phenotyping to characterize patients, develop targeted treatment regimens, and assess the efficacy of therapies for mild TBI.

Therapies that appear promising in pre-clinical testing are more likely to translate to successful clinical trials if they result in improvements in behavioral outcomes that are relevant to humans. Shultz and colleagues summarize the behavioral consequences of TBI in humans and describe behavioral tasks appropriate for experimental animals that measure the functional outcomes that are most often assessed in patients with TBI. Additionally, they provide an overview of the results of studies using clinically relevant behavioral tasks in experimental models of TBI, focusing on preclinical behavior methods and findings in terms of their relevance to studies in patients with TBI. Finally, they outline strategies and future research to improve the clinical relevance of behavior testing in animal models of TBI.

Poloyac and colleagues describe the pharmacological barriers to the successful development of drug therapies for TBI and discuss strategies to overcome these barriers. They review pharmacological considerations for moving from disease targets to lead compounds with drug-like and central nervous system (CNS) penetrant properties. The authors describe the use of in vitro assessments of druglike properties, followed by pre-clinical studies in vivo to ensure accurate identification of the pharmacokinetic and pharmacodynamic characteristics of response. Poloyac and colleagues highlight the importance of biomarker development and utilization in preclinical and clinical studies as well as the importance of the identification of diagnostic, pharmacodynamic/response and prognostic biomarkers of injury type or severity, target engagement, and disease progression. Their review describes important considerations in determining pre-clinical dose selection in vivo and cross-species and human equivalent dose selection and the use of allometric scaling, and pharmacokinetic and pharmacodynamic criteria for clinical trial design.

As noted above, one of the potential reasons for the lack of successful translation of promising pre-clinical therapies is the limited use of large animals in pre-clinical testing. To address this problem, Armstead and colleagues describe the use of reverse translation of bedside experience to improve the design of preclinical studies in a porcine model of TBI that accurately replicates practical clinical assessments. In patients with TBI, cerebral perfusion pressure (CPP) is often normalized using vasoactive agents to increase mean arterial pressure (MAP) and counteract the effects of impaired cerebral autoregulation and improve neurological outcomes. However, to date, the effects of many of these vasoactive agents (e.g., phenylephrine, dopamine, norepinephrine, and epinephrine) on CPP, autoregulation, and survival after TBI have not been thoroughly explored. Armstead and colleagues describe a bidirectional translational approach using a clinically relevant large-animal model of TBI to better identify therapeutic strategies designed to improve outcome post-injury.

Issadore and colleagues describe their open-ended search for panels of microRNA (miRNA) biomarkers contained in extracellular vesicles (EVs) that will more accurately classify various states of injury. They analyzed samples from experimental studies using a variety of murine injury types, injury intensities, history of injuries, and times post-injury, as well as samples from patients with TBI.

Department of Anesthesiology, University of Texas Medical Branch, Galveston, Texas, USA. 
Using next-generation sequencing coupled with Track Etched Magnetic Nanopore (TENPO) sorting, a technique developed in their laboratories, they enriched the GluR2+EVs and profiled their miRNA. These innovative methods resulted in the mapping and comparing of brain-derived EV miRNA between various injuries, thereby identifying signaling pathways that connect these biomarkers to underlying mechanisms of TBI in the pre-clinical model and the clinical samples. They then applied machine learning to define a panel of biomarkers to successfully classify specific states of injury, paving the way for a prognostic blood test for TBI.

Glushakova and colleagues observed that, although intracranial pressure (ICP) is one of the most common neurologically specific physiological variables used to direct the care of patients with severe TBI (sTBI). Recent clinical evidence has called into question the association of ICP monitoring with improved clinical outcome. The specific cellular and molecular derangements associated with intracranial hypertension (IC-HTN) and their relationship to neurological outcomes are not well understood. Additionally, despite decades of research using a variety of experimental TBI models, the clinical applicability of ICP monitoring in the pre-clinical setting has not been established. Glushakova and colleagues suggest that the linking of basic mechanistic studies in TBI models with investigations of ICP monitoring that more accurately replicate the clinical setting will provide clinicians with a better understanding of the pathophysiology of IC-HTN, thus facilitating development of improved therapies for patients with sTBI.

Hawkins and colleagues suggest that TBI translation is a problem of big-data that can be best addressed using modern data science approaches. They summarize the history of the term "big-data," which originated in Internet technology as data that are big according to the four "Vs" of volume, velocity, variety, or veracity, and then transitioned into the mainstream of biomedical research. They suggest that TBI translation fundamentally involves data variety, a problem that could best be addressed using modern machine learning and other cutting-edge analytical approaches. Further, they discuss the importance of acquiring data from diverse sources including unpublished sources (i.e., "dark data") and long-tail data (small, specialty TBI data sets undergirding the published literature). ${ }^{14}$ Finally, they summarize the results of published articles describing pre-clinical and clinical TBI research to suggest ways that data reuse can drive new discoveries leading to successful translation of TBI therapies. Ideally, these methods will facilitate the development of TBI data resources that are more Findable, Accessible, Interoperable, and Reusable (FAIR) to accelerate discovery and translation for the silent epidemic of TBI.

\section{References}

1. Bragge, P., Synnot, A., Maas, A.I., Menon, D.K., Cooper, D.J., Rosenfeld, J.V., and Gruen, R.L. (2016). A state-of-the-science overview of randomized controlled trials evaluating acute management of moderate-to-severe traumatic brain injury. J. Neurotrauma $33,1461-1478$.
2. Burke, M.J., Fralick, M., Nejatbakhsh, N., Tartaglia, M.C., and Tator, C.H. (2015). In search of evidence-based treatment for concussion: characteristics of current clinical trials. Brain Inj. 29, 300-305.

3. Marklund, N., Bakshi, A., Castelbuono, D.J., Conte, V., and McIntosh, T. (2006). Evaluation of pharmacological treatment strategies in traumatic brain injury. Curr. Pharm. Des. 12, 1645-1680.

4. Agoston, D.V., Risling, M., and Bellander, B.-M. (2012). Bench-tobedside and bedside back to the bench; coordinating clinical and experimental traumatic brain injury studies. Front. Neurol. 3, 1-5.

5. Kochanek, P.M., Bramlett, H.M., Dixon, C.E., Shear, D.A., Dietrich, W.D., Schmid, K.E., Mondello, S., Wang, K.K.W., Hayes, R.L., Povlishock, J.T., and Tortella, F.C. (2016). Approach to modeling, therapy evaluation, drug selection, and biomarker assessments for a multicenter pre-clinical drug screening consortium for acute therapies in severe traumatic brain injury: Operation Brain Trauma Therapy. J. Neurotrauma 33, 513-522.

6. Loane, D.J., and Faden, A.I. (2010). Neuroprotection for traumatic brain injury: translational challenges and emerging therapeutic strategies. Trends Pharmacol. Sci. 31, 596-604.

7. Page, M.J., Higgins, J.P., Clayton, G., Sterne, J.A.C., Hrobjartsson, A., and Savovic, J. (2016). Empirical evidence of study design biases in randomized trials: systematic review of meta-epidemiological studies. PLoS One 11, e0159267.

8. Button, K.S., Ioannidis, J.P.A., Mokrysz, C., Nosek, B.A., Flint, J., Robinson, E.S.J., and Munafo, M.R. (2013). Power failure: why small sample size undermines the reliability of neuroscience. Nat. Rev. Neurosci. 14, 365-376.

9. Egger, M., Davey-Smith, G., Schneider, M., and Minder, C. (1997). Bias in meta-analysis detected by a simple, graphical test. Brit. Med. J. 315, 629-634.

10. Hawkyrluk, G.W.J., and Bullock, M.R. (2015). Design of acute neuroprotection studies, in: Handbook of Clinical Neurology, Traumatic Brain Injury, Part II, Vol. 128, J. Grafman, and A.M. Salazar (eds). ScienceDirect, pps. 761-778.

11. Menon, D.K. (2009). Unique challenges in clinical trials in traumatic brain injury. Crit. Care Med. 37, S129-S135.

12. Stocchetti, N., Taccone, F.S., Citerio, G., Pepe, P.E., Le Roux, P.D., Oddo, M., Polderman, K.H., Stevens, R.D., Barsan, W., Maas, A.I., Meyfroidt, G., Bell, M.J., Silbergleit, R., Vespa, P.M., Faden, A.I., Helbok, R., Tisherman, S., Zanier, E.R., Valenzuela, T., Wendon, J., Menon, D.K., and Vincent, J.L. (2015). Neuroprotection in acute brain injury: an up- to-date review. Crit. Care 19, 186.

13. DeWitt, D.S., Hawkins, B.E., Dixon, C.E., Kochanek, P.M., Armstead, W., Bass, C.R., Bramlett, H.M., Buki, A., Dietrich, W.D., Ferguson, A.R., Hall, E.D., Hayes, R.L., Hinds, S.R., LaPlaca, M.C., Long, J.B., Meaney, D.F., Mondello, S., Noble-Haeusslein, L.J., Poloyac, S.M., Prough, D.S., Robertson, C.S., Saatman, K.E., Shultz, S.R., Shear, D.A., Smith, D.H., Valadka, A.B., VandeVord, P., and Zhang, L. (2018). Preclinical testing of therapies for traumatic brain injury. J. Neurotrauma 35, 2737-2754.

14. Ferguson, A.R., Nielson, J.L., Cragin, M.H., Bandrowski, A., and Martone, M.E. (2014). Big data from small data: data-sharing in the 'long tail' of neuroscience. Nat. Neurosci. 17, 1442-1448.

Address correspondence to: Douglas DeWitt, PhD Department of Anesthesiology University of Texas Medical Branch 301 University Boulevard, Suite 2A Galveston, TX 77555 USA

E-mail: ddewitt@utmb.edu 